\title{
"Moonlighting" adhesins at the cell surface of Candida glabrata pathogenic yeasts
}

\author{
A. Kozik1, D. Satala1, O. Bochenska1, J. Karkowska-Kuleta², M. Rapala-Kozik²
}

${ }^{1}$ Department of Analytical Biochemistry, ${ }^{2}$ Department of Comparative Biochemistry and Bioanalytics;

Faculty of Biochemistry, Biophysics and Biotechnology, Jagiellonian University in Krakow, Krakow, Poland

The adhesion of microbial pathogens to the host cells-a critical step for the host infection-depends on an arsenal of proteins, collectively called adhesins that are exposed on the pathogen surface and bind numerous host proteins. Besides typical adhesins, some pathogen proteins are loosely associated with the cell surface and identical with well known intracellular enzymes, involved in the crucial conserved metabolic routes. Secreted via yet unknown mechanism(s), these "moonlighting proteins" perform a new function of adhesins. Candida glabrata is a common fungal pathogen of humans. Unlike other species from the Candida genus, $C$. glabrata is haploid yeast unable to convert into invasive hyphal forms, but still possesses a wide repertoire of virulence attributes, including the presence of adhesive proteins at the fungal cell surface.

The present study aimed to identify C. glabrata

surface-exposed proteins that can be classified as moonlighting proteins with a putative adhesin activity.

RESULTS: SURFACE PLASMON RESONANCE ANALYSIS OF FIBRONECTIN AND VITRONECTIN BINDING
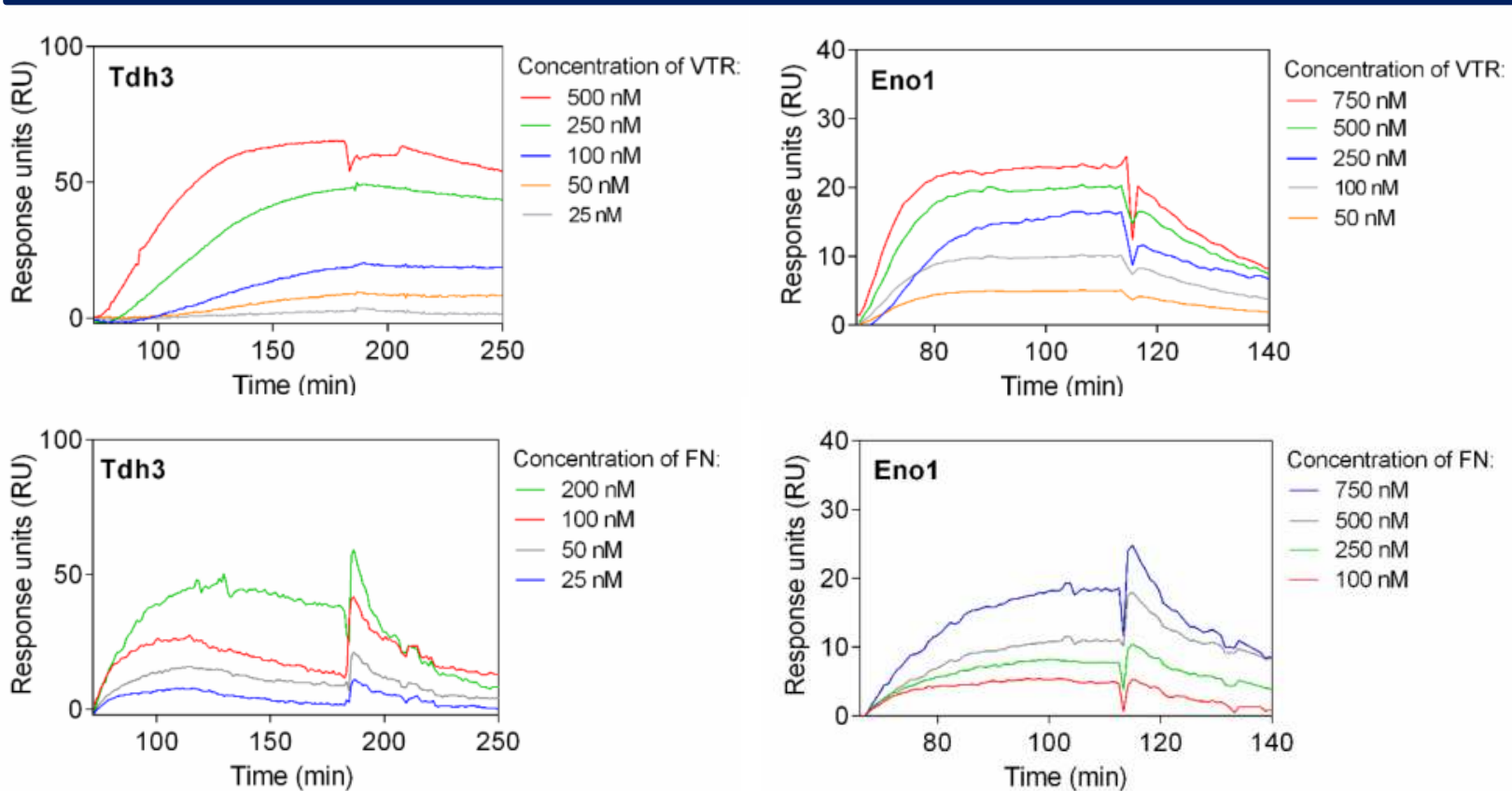

\begin{tabular}{|c|c|c|c|c|}
\hline \multirow{2}{*}{} & \multicolumn{2}{|c|}{ Tdh3 } & \multicolumn{2}{c|}{ Eno1 } \\
\cline { 2 - 5 } & VTR & FN & VTR & FN \\
\hline $\mathrm{KD}[\mathrm{M}]$ & $4,24 \times 10^{-8}$ & $5,76 \times 10^{-8}$ & $5,35 \times 10^{-8}$ & $1,78 \times 10^{-7}$ \\
\hline $\mathrm{ka}\left[1 / \mathrm{M}^{*} \mathrm{~s}\right]$ & $4,20 \times 10^{4}$ & $2,24 \times 10^{5}$ & $4,54 \times 10^{5}$ & $1,65 \times 10^{5}$ \\
\hline $\mathrm{kd}[1 / \mathrm{s}]$ & $1,78 \times 10^{-3}$ & $1,29 \times 10^{-2}$ & $2,42 \times 10^{-2}$ & $2,93 \times 10^{-2}$ \\
\hline
\end{tabular}

Figure 1. Kinetic and thermodynamic parameters of VTR and FN binding by $C$. glabrata moonlighting proteins-Tdh3 and Eno1determined by surface plasmon resonance measurements (SPR). The binding parameters for interactions between fungal proteins (ligands) and VTR or FN (analytes) were determined after fitting the data with a simple Langmuir model (1:1) with drifting baseline and Rmax locality.

Conclusions A large number of various surface-exposed proteins were detected, including typical cell wall proteins and a wide variety of moonlighting proteins. The dissociation constants determined by SPR measurements for the complexes of Eno1 and Tdh3 with human vitronectin and fibronectin, suggests their adhesin function. The presence of numerous diverse moonlighting proteins at the surface of $C$. glabrata cells may indicate their potential collective involvement in the pathogenesis of infections caused by these yeasts.
Methods C. glabrata was cultured in various media-vaginasimulative medium (VS) and basal medium mucin (BMM). Yeast cells cultured in synthetic Lee's medium were served as a control. The fungal "surfaceome" was analyzed by a rapid method based on the "cell surface shaving" with trypsin, combined with the "shotgun" proteomic approach. Some of the moonlighting proteins-Eno1 and Tdh3-were isolated from a fraction of beta-1,6-glucanase-extractable cell-wall proteins and purified by ion-exchange and gel filtration chromatography. Their ability to bind human extracellular matrix proteins-fibronectin (FN) and vitronectin (VTR)-were quantitatively characterized using the surface plasmon resonance (SPR) measurements.

\section{RESULTS: CELL SURFACE SHAVING WITH TRYPSIN - IDENTIFICATION OF C. GLABRATA CELL SURFACE- EXPOSED PROTEINS}

Table 1. Changes in major protein levels in C. glabrata in response to different growth conditions compared to the synthetic Lee's medium. After peptide identification using LC-MS/MS a final dataset was created on the basis of the classical mathematical normalization, named the normalized spectral abundance factor (NSAF) that allows to estimate the relative protein abundances and calculate the intensities of changes.

\begin{tabular}{|c|c|c|c|}
\hline \multirow[b]{2}{*}{ Accession } & \multirow[b]{2}{*}{ Protein } & \multicolumn{2}{|c|}{ Level of changes } \\
\hline & & $\begin{array}{c}\text { Medium } \\
\text { BMM }\end{array}$ & $\begin{array}{c}\text { Medium } \\
\text { VS }\end{array}$ \\
\hline XP_446770.1 & $\begin{array}{l}\text { glyceraldehyde-3-phosphate } \\
\text { dehydrogenase (Tdh3) }\end{array}$ & 0.7 -fold & 2.5 -fold ${ }^{\uparrow}$ \\
\hline XP 447969.1 & secreted glycoprotein (Ygp1) & 1 -fold $\downarrow$ & 2.5 -fold ${ }^{\uparrow}$ \\
\hline XP 447360.1 & enolase I (Eno1) & 2-fold $\uparrow$ & 4-fold $\uparrow$ \\
\hline XP_449113.1 & phosphoglycerate kinase (Pgk1) & $\leftrightarrows$ & \\
\hline XP 448795.1 & heat shock protein (Hsp82) & 2.5 -fold $\uparrow$ & \\
\hline XP_445764.1 & $\begin{array}{c}\text { similar to yapsin (Yps3) Saccharomyces } \\
\text { cerevisiae }\end{array}$ & $\leftrightarrows$ & \\
\hline XP 446378.1 & soluble cell wall protein (Scw4) & 2.5 -fold $\uparrow^{\uparrow}$ & 5 -fold $\uparrow$ \\
\hline XP 445106.1 & transaldolase (Tal1) & 2-fold $\uparrow$ & \\
\hline AF_545432.1 & pyruvate decarboxylase (Pdc) & & $\uparrow \uparrow$ \\
\hline$X P \_445189.1$ & epithelial adhesin 6 (Epa6) & & $\uparrow \uparrow$ \\
\hline XP_446298.1 & cell wall mannoprotein (Cwp1) & $\uparrow \uparrow$ & \\
\hline$X P \_447033.1$ & phosphoglucose isomerase (Pgi) & $\uparrow \uparrow$ & \\
\hline$X P \_447161.1$ & triosephosphate isomerase (Tpi) & $\uparrow \uparrow$ & \\
\hline$X P \_447518.1$ & beta-1,3-glucan linked protein (Pir1) & $4 \uparrow$ & \\
\hline$X P \_448549.1$ & trehalose-phosphatase (Tps) & & $\uparrow \uparrow$ \\
\hline
\end{tabular}

$\uparrow$ increase; $\downarrow$ decrease; $\leftrightarrows$ no changes; $\uparrow \uparrow$ in VS or BMM only
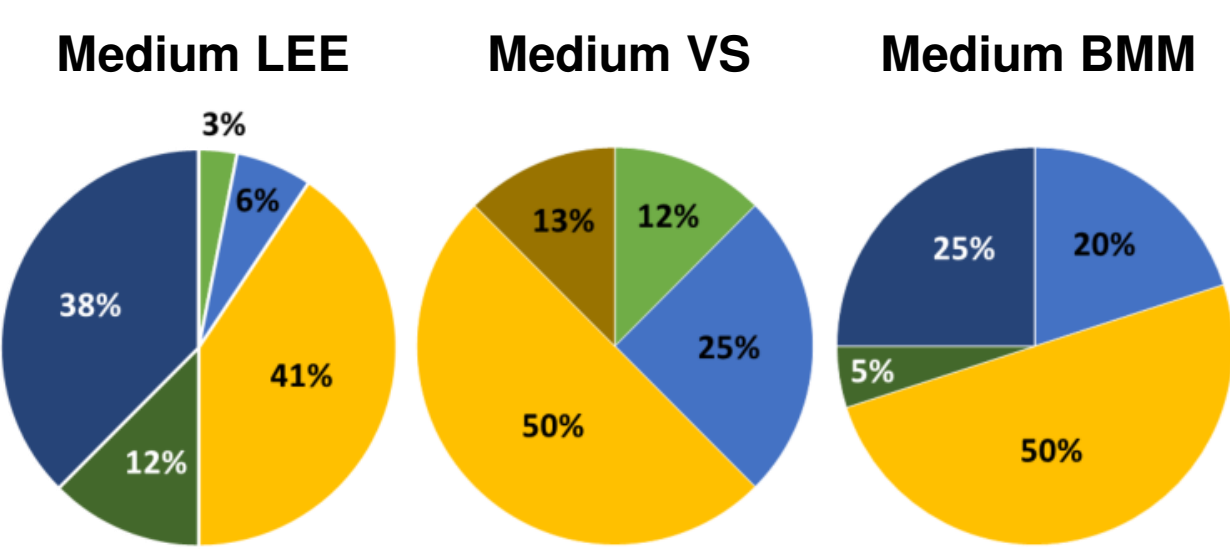

madhesion

- cell wall maintenance

- sterss response

- protein synthesis

metabolism

- unknown function

Fiqure 2. Functional classification of $C$. glabrata surface-exposed proteins identified with the cell surface shaving method and mass spectrometry. Protein functions have been assigned on the basis of descriptions from NCBI protein database and orthologs' description in the Candida Genome Database. 